\title{
Noise Reduction in Spatial Data using Machine Learning Methods for Road Condition Data
}

\author{
Dara Anitha Kumari ${ }^{1}$ \\ CSE Department, JNTUH CEH \\ Hyderabad, Telangana, India
}

\author{
Dr. A. Govardhan ${ }^{2}$ \\ CSE Department, JNTUH \\ Hyderabad, Telangana, India
}

\begin{abstract}
With the increase in the road transportation system the safety concerns for the road travels are also increasing. In order to ensure the road safety, various government and non-government efforts are visible to maintain the road quality and transport network system. The maintenance of the road condition is in the verse of getting automated for the quick identification of potholes, cracks and patch works and repair. The automation process is taking place in majority of the counties with the help of ICT enabled frameworks and devices. The primary device used for the purpose is the geo location enabled image capture devices. Regardless to mention the image capture process is always prune to noises and must be removed for better further analysis. Also, the spatial data is collected from the road networks are also prune to various error such as missing values or outliers due to the induced noises in the capture devices. Hence, the demand of the current research is to purpose a complete solution for the noise identification and removal from the spatial road network data for making the automation process highly successful and highly accurate. In the recent time, many parallel research attempts are observed, which resulted into solving the problem of noise reduction in all aspects of spatial data. Nevertheless, all the parallel research outcomes have failed to provide a single solution for all the noise issues. Henceforth, this work proposes three novel algorithms to solve spatial image noise problem using the adaptive moment filtration, missing value noise from the spatial data using adaptive logistic analysis and finally, the outlier noise removal from the same spatial data using corrective logistic machine learning method. The outcome of this work is nearly $\mathbf{7 0 \%}$ accuracy in image noise reduction, 90\% accuracy for missing value and outlier removal. The work also justifies the information loss reduction by nearly $50 \%$. The final outcome of the work is to ensure higher accuracy for road maintenance automation.
\end{abstract}

Keywords-Spatial image moments; adaptive logistic denoising; machine learning; noise removal; correlative corrections

\section{INTRODUCTION}

Spatial data retrieval for the imaging methods are highly beneficial for detection and provide automatic maintenance of the road conditions as the spatial data provides higher order information for each pixel in the spatial image. The work by D. A. Landgrebe [1] have significantly proven the fact the improvement in further processing can be achieved using spatial data. Nonetheless, the accumulation of the spatial image data is full of challenges as suggested by J. M. Bioucas - Dias [2] with the restriction of continuous mapping between image data and spatial data vectors. The other parallel research by $\mathrm{N}$. Keshava [3] have suggested various other methods for mapping without the spatial mixing methods.
However, there are multiple parallel research outcomes, which suggested extraction of the spatial data by separating the image information and the text information associated with each pixel. The study by M. E. Winter [4] Have suggest the extraction of the road spatial data using the N-Finder algorithm. This algorithm is sophisticated and highly accepted by the researcher for spatial data extraction using the vector methods. Also, the work by J. M. P. Nascimento [5] have demonstrated the vertex component analysis method, which relies on the modifications and enhancements over the principle component extraction methods. Further, the work by J. Li [6] has showcased the algorithm as minimum volume measure, ensuring the extraction of the minimal spatial data for any purpose such as road information.

Nonetheless, having the capture process sorted out for the spatial data accumulation, the major focus is on the noise reduction as suggested by Alp Erturk [7]. Henceforth, this work proposes a noval solution for spatial data noise reduction for all possible noise types.

The rest of the work is furnished such that in Section 2, the parallel research outcomes are analysed, in Section 3, the mathematical model for the identified problems and the proposed solutions are furnished, in Section 4 the proposed algorithm are elaborated, in Section 5, the obtained results are discussed, in Section 6 the comparative analysis is summarized and the work produces the final conclusion in Section 7.

\section{PARALlEL RESEARCH OUtCOMES}

In the section of the work, the parallel research outcomes are discussed critically for better formulation of the problem and solutions in the upcoming sections.

The noise in any form in any data can be catastrophic for achieving the further results. Hence, the pre-processing techniques for the data noise reductions are getting popular. However, the present pre-processing method for the spatial data is not highly complex and demands further improvements. When there is significantly immaterial and redundant and data present or noisy and unreliable data, then comprehension discovery during the training period is more difficult steps that are filtering and data preparation may require considerable level of processing period. Data preprocessing comprises cleansing, Instance selection, normalization, transformation, characteristic extraction along with variety, etc. 
The recent research by M.Zortea [8] have pro-posed a spatial pre-processing method for image data using endmember extraction process. The work is highly justified for the pixel information preservation and noise reduction at the same time. Nonetheless, the associated information extracted from the pixels are also prune to noises, which must be de-noised as well. However, the work by G.Martin [9] denoising process, where the relevancy of the information is also considered. This ensures some of the extracted information from te pixels are also processes for normalization. Further, the work of A. Plaza [10] has fine-tuned the workability of the spatial image information de-noising by applying the remotely sensed hyperspectral analysis for the spatial datasets.

In the contrast, the work by A. Erturk [11] and D. Cesmeci [12] has demonstrated significant contributions to the denoising of the spatial data. These two works are highly accepted. Nevertheless, this work identified few drawbacks in these two bench-marked algorithms and proposes a solution to these methods. In the further sections of the work, these two works are constantly compared with the proposed outcomes. Finally, the similar problems are also highlighted by the work of C. Li [13].

The limitations of the existing research outcomes are listed here: Firstly, all signal processing apparatus, both analogy along with electronic, have faculties which make them more prone to noise. Noise might be arbitrary or white sound using an additional frequency supply, or frequency determined noise introduced with means of a computer device's mechanism or indicate processing calculations.

Secondly, back in electronic recording apparatus, a significant kind of noise can be hiss created by arbitrary electron motion due to thermal agitation in any way temperatures above absolute zero. These abrasive electrons quickly add and subtract out of the voltage of this output and therefore create detectable sound.

Finally, in the instance of photographic picture along with magnetic tape is introduced on account of the grain structure of this moderate. In photographic picture, the magnitude of the sausage at the film determines the picture's sensitivity, more sensitive picture with larger sized proteins. To pay for this, larger regions of film or magnetic tape could be employed to lessen the noise to a decent level.

Henceforth, in the next section of the work, the problems are highlighted, elaborated and the mathematical models for the proposed solutions are formulated.

\section{PROBLEM ForMULATION}

After the detailed analysis of the parallel research outcomes, in the section of the work, the mathematical model for the problem and proposed solutions are elaborated.

Lemma-1: Adaptive Moment Filtration can detect and reduce the noise in the spatial image sets.

Proof: Assuming that the complete spatial image ste can be represented as I[] and each and every image in the set can be identified as $I_{x}$ with a total number of $n$ images in the dataset. Then, this analogy can be represented as:
$I[] \leftarrow \sum_{x=1}^{n} I_{x}$

Where each $I_{x}$ belongs to the spatial dataset. As,

$I_{x} \in I[]$

Also, every image in the dataset can be represented using the $<\mathrm{r}, \mathrm{g}, \mathrm{b}>$ tuple as,

$I_{x}=<r_{x}, g_{x}, b_{x}>$

Applying, the $\phi$ function for extraction of the gray parameter as intensity of the image as $g_{x}$

$\phi\left(I_{x}\right) \rightarrow g_{x}$

Henceforth the complete image can be clustered based on the adaptive image intensity. Assuming the cluster collection for the image as $\mathrm{C}$ [], the following formulation can be derived:

$$
C[]=\sum_{i, j=1}^{n . m} \frac{\left(g_{i}-g_{i+1}\right)^{n} \cdot\left(g_{j}+g_{j-1}\right)^{m}}{\left\{\left(g_{j-1}-g_{j}\right)+\left(g_{j+1}-g_{j}\right)+\left(g_{i-1}-g_{i}\right)+\left(g_{i+1}-g_{i}\right)\right\}}
$$

Further, assuming that, each and every cluster in the cluster set $\mathrm{C}[]$ can be represented in terms of two dimensional function to map into the spatial space, then this concept can be mathematical presented as,

$$
C_{k} \in C[]
$$

And,

$$
C_{k}=\{f(i, j) \mid(m, n)\}
$$

Where $\mathrm{m}$ and $\mathrm{n}$ are the order of the spatial data. Thus the moment $\mathrm{M}_{\mathrm{k}}$, can be calculated as,

$$
M_{k}=\int_{-\infty}^{+\infty} \int_{-\infty}^{+\infty} i^{m} j^{n} \cdot f(i, j) \cdot d i \cdot d j
$$

Similarity, for the complete image, the same moment function can be calculated as

$$
M_{I}=\int_{-\infty}^{+\infty} \int_{-\infty}^{+\infty} i^{\prime m^{\prime}} j^{\prime^{\prime}} \cdot f\left(i^{\prime}, j^{\prime}\right) \cdot d i^{\prime} \cdot d j^{\prime}
$$

Where,

$$
C_{I}=\left\{f\left(i^{\prime}, j^{\prime}\right) \mid\left(m^{\prime}, n^{\prime}\right)\right\}
$$

Finally, building the complete set of clusters with noises $\mathrm{CN}[]$, each and every cluster must be analysis as,

$$
C N[]=\forall C_{k} \in C[], \text { iff } M_{k} \neq M_{I}
$$


Assuming that each cluster in the image set with noise can be represented as $\mathrm{CN}_{n}$, then each $\mathrm{CN}_{n}$ must be clustered again using the adaptive clustering process and the moments shall be calculated, considering only the selected cluster.

Considering the final size of the sub-cluster is relatively small considering the complete image, the sub-cluster with the noise can be replaced using the adaptive missing value replacement method. This adaptive missing value replacement method is further elaborated in the text mathematical model in this section.

Lemma -2: Adaptive logistic analysis for the missing value can be highly accurate compared with the linear analysis for spatial data.

Proof: Assuming that, the spatial dataset can be considered as D [] and each item in the data set can be represented as $D_{x}$ with total number of elements as $n$ with total $m$ number of tuples. Thus, this relation can be formulated as,

$D[]=\frac{n}{\sum_{x=1}^{m}<D_{x}, D_{x}^{\prime}, D_{x}^{\prime \prime} \ldots>}$

The linear method applies a simple strategy for calculating the replacement factor, RF [] as,

$R F[]=\sum_{x=1}^{n^{m}}\left|D_{x+1}-D_{x}\right|$

Henceforth, the men of the RF [], denoted as rf, can be calculated as,

$r f=\frac{\sum_{i=1}^{n^{m}} R F[i]}{\delta(R F[])}$

In the other hand, the adaptive logistic analysis builds the correlation factor, Corr, before calculating the rectification factor as,

$\operatorname{Corr}(t+1)=\sum_{x=1}^{n^{m}} \frac{\partial^{2}\left\{\ln \left(D_{x+1}\right)-\frac{\ln \left(D_{x}\right)}{\Delta \operatorname{Corr}(t)}\right\}}{\partial \frac{\ln \left(D_{x+1}\right)^{2}}{\Delta \operatorname{Corr}(t)}}$

Further, the rectification factor, rf, can be calculated as,

$r f^{\prime}=\frac{\sum_{t=1}^{n^{m}} \operatorname{Corr}(t)}{\delta(\operatorname{Corr}[])}$

It is natural to realize that, the adaptive logistic method is prone to be a lesser number compared to the linear method [Fig. 1], Hence, the following statement can be made, as $r f^{\prime}<<r f$

Henceforth, if any data point can be featured as missing value data point in the spatial dataset,

$D_{x} \rightarrow(N / A) \rightarrow 0$

And

$D_{x} \notin D_{x-1} \pm \operatorname{Corr}[]$

Then, using the adaptive logistic method, the missing data point can be calculated as,

$D_{x}(t+1)=D_{x-1}(t) \pm \operatorname{Corr}[t]$

Thus, it is realist to the state that, adaptive logistic method is more accurate for identifying and replacing the missing values or data points in the spatial datasets.

Furthermore, the final identified challenge for the spatial data noise reduction is the outlier removal from the outlier form dataset or data points. Hence, this problem must be addressed and the solution to this problem is formulated using the following mathematical model.

Lemma -3 Corrective logistic analysis for the outlier value can be accurate compared with the linear analysis for spatial data.

Proof: Assuming that, the spatial dataset can be considered as D [] and each time in the data set can be represented as $D_{x}$ with total number of elements as $n$ with total $m$ number of tuples. Thus, this relation can be formulated as,

$$
D[]=\frac{n}{\sum_{x=1}^{m}<D_{x}, D_{x}^{\prime}, D_{x}^{\prime \prime} \ldots>}
$$

The liner method applies a simple strategy for calculating the replacement factor, RF [] as,

$$
R F[]=\sum_{x=1}^{n^{m}}\left|D_{x+1}-D_{x}\right|
$$

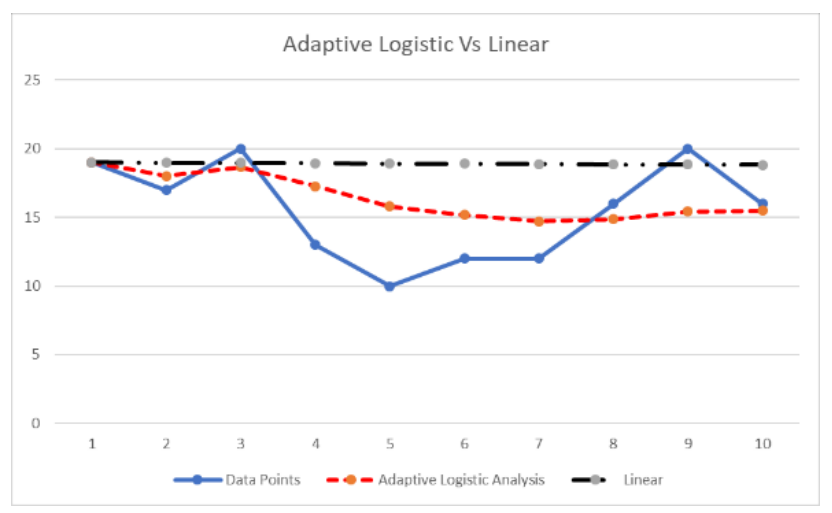

Fig. 1. Comparative Function Growth in Adaptive Logistic and Linear. 
Henceforth, the mean of the RF [], denoted as rf, can be calculated as

$r f=\frac{\sum_{i=1}^{n^{m}} R F[i]}{\delta(R F[])}$

In the other hand, the adaptive logistic analysis builds the threshold factor, $\mathrm{TH}$, as,

$T H(t)=\frac{\sum_{i=0}^{n} \ln \left(D_{x}\right)^{x}}{\partial t} \pm \theta(t)$

Further, the correction factor, $\theta(t)$, can be calculated as,

$\theta(t)=\frac{\theta(t-1)}{\partial \theta[]}$

Again, it is natural to realize that corrective logistic method is prone to be a lesser number compared to the linear method and can be formulated as

$T H<<r f$

Henceforth, if any data point can be featured as outlier data points in the spatial dataset,

$D_{x}(t) \rightarrow T H(t+1)$

Then, the outlier data point must be replaced with the newer data point as,

$D_{x}(t) \rightarrow T H(t) \pm \theta(t)$

Thus, it is realist to state that, corrective logistic method is more accurate for identifying and replacing the outliers' values or data points in the spatial datasets.

Henceforth, in the light of the problem formulation and proposed corrective models, in the next section of this work, the proposed algorithms are furnished and discussed.

\section{PROPOSED ALGORITHMS}

After the detailed mathematical modelling of the problem and proposed solution, in this section of the work, the proposed algorithms are furnished and discussed.

Firstly, the algorithm for noise detection and reduction is furnished here for the spatial images.

Moments are well-known for his or her own application in picture investigation, because they are sometimes utilized to derive invariants with regard to special conversion lessons. The expression invariant minutes can be abused within this circumstance. But whilst instant invariants are all invariants which can be made by minutes, the single minutes which can be invariants on their own would be the fundamental minutes. Be aware the invariants will be invariant from the domain names. Neither scaling nor spinning have been characterized also the transformation is not reversible, and also an image is an approximation. All these invariants are simply invariant after describing a silhouette.

Secondly, the algorithm for missing value reduction for spatial data is furnished here.

Algorithm - I: Adaptive Moment Based Spatial Image Noise Detection and Removal Algorithm (AMBSI-NDR)

Step - 1. Accept the spatial data set V [].

Step - 2. For each V [i] in V[]

a. Separate the text component as T[i] and Image Components as I[i].

b. For each $\mathrm{T}[\mathrm{i}]$ in $\mathrm{T}[]$

i. Call the ALC-MVIR and CLC-OIR algorithms.

c. For each $\mathrm{I}[\mathrm{k}]$ in $\mathrm{I}[]$

i. Calculate the Pixel Intensity for all Pixels, PI [].

ii. Calculate the Initial Noise Level as NI.

iii. Calculate the Image Size as IS.

iv. For each PI[i] in PI[]

1. Consider the Image as ImgTem.

2. Identify the Image Segments as $\mathrm{S}[]$.

v. Calculate the Image Moment as IM.

vi. For each $\mathrm{S}[\mathrm{i}]$ in $\mathrm{S}[]$

1. Calculate the moment as $\mathrm{M}[\mathrm{i}]$

2. If $\mathrm{M}[\mathrm{i}]$ Not Equals to IM

a. $\quad$ Then, $\operatorname{ImgTem}=\mathrm{S}[\mathrm{i}]$, $\mathrm{IM}=\mathrm{M}[\mathrm{i}]$ and Repeat from Step-2.C.I

3. Else, Mark the S[i] as NoNoise

vii. Replace the Segments Marked NoNoise data using CLC-OIR Algorithm

d. Calculate the Reduced Noise Level as RNI.

e. Calculate the Final Image Size as FIS.

f. If RNI < NI and FIS = IS

i. Then, Report the Final Image without Noise.

g. Else, Repeat from Step-2.C.

Step - 3. Report the final noise reduced image set I[]. 
Imputation could be the procedure for substituting lost data using values. It's called thing imputation when it's called imputation. When substituting for a factor of the data level. Now, there are three major difficulties that data will cause data make reductions, create the analysis and handling of their data arduous, and may present a sizable total of prejudice.

Algorithm - II: Adaptive Logistic Correlation Based
Missing Value Identification \& Replacement
Algorithm (ALC-MVIR)
Step - 1. Accept the Text set as T[] from the Spatial
Dataset
Step - 2. For each T[i] from T[] set
a. Calculate the Correlation, Corr, for each
Data Iteams as Eq. 15
Calculate the rectification factor, Corr as
Eq. 16
If T[i] Equals to Zero or T[i] Equals to
"Not Available"
Then,
i. Mark the data items or Data
ii. Replace the Missing Value as
Step - 3. Report the final Missing Value Reduced
dataset as T[]

Finally, the algorithm for outlier detection in spatial data sets are furnished here.

Algorithm - III: Correlative Logistic Correction Based Outlier Identification \& Removal Algorithm (CLC-OIR)

Step-1. Accept the Text set as T[] from the Spatial Dataset

Step - 2. For each T[i] from T[] set
a. Calculate the Threshold, TH[i] as Eq. 24
b. Calculate the correction factor, Theta[i] as Eq. 25
c. If $\mathrm{T}[\mathrm{i}]>\mathrm{TH}[\mathrm{i}]$
d. Then,

i. Mark the data item or Data Point as Outlier

ii. Replace the Outlier as $\mathrm{T}[\mathrm{i}]=\mathrm{TH}[\mathrm{i}]$ \pm Theta[i]

Step - 3. Report the final outlier removed dataset as T[]

They suggest either dimension error or the people come with a supply, although outliers can happen by chance in virtually and supply. At the prior event one wants to lose them use statistics which can be robust to outliers, any particular you must be careful in applying and then although at the latter instance that they signify which the supply comes with skewness. A reason for outliers can be that a mix of two distributions, which could signal identification versus dimension malfunction, or maybe just two, means of mix version model this really.

The results obtained from the proposed algorithms are highly satisfactory and are discussed in the next section of the work.

\section{RESULTS AND DISCUSSIONS}

The proposed algorithm is evaluated on standard and benchmark dataset [14] and are highly satisfactory. In the section of the work, the obtained results are furnished and discussed.

Firstly, the image sets are introduced with some additional noises for better identification of the improvements over the traditional algorithms with the proposed algorithm. The initial noise induction results are elaborated here [Table I]. The noise types induced as Type-1 for Salt and Pepper Noise, Type-2 for Gaussian Noise, Type-3 for Sparkle Noise and finally Type-4 for passion Noise.

The noise levels are also visualized graphically here [Fig. 2].

Secondly, the results from the image noise reduction algorithm is furnished [Table II] and the step by step comparison is also done with two benchmark work by A. Erturk [11] and D. Cesmeci [12].

The results are also visualized graphically here [Fig. 3].

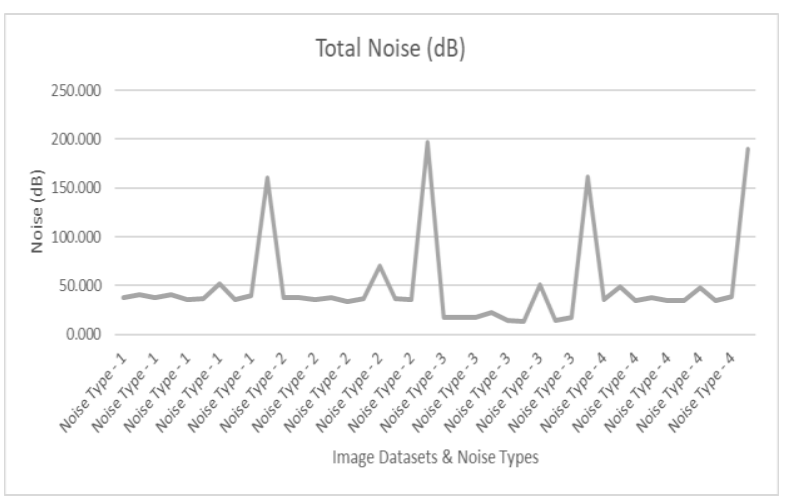

Fig. 2. Initial Noise Level Analysis.

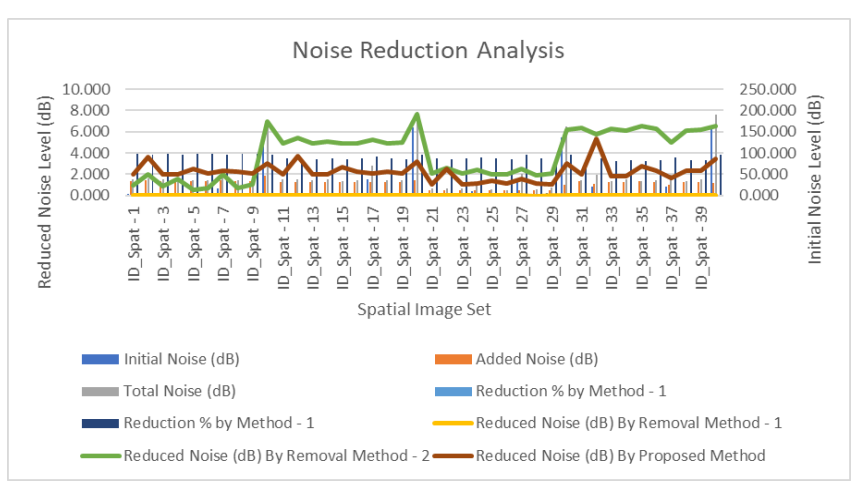

Fig. 3. Noise Level Reduction Analysis. 
TABLE. I. NOISE INDUCTION IN SPATIAL IMAGES

\begin{tabular}{|c|c|c|c|c|}
\hline & Data Item & $\begin{array}{l}\text { Initial } \\
\text { Noise } \\
\text { (dB) }\end{array}$ & $\begin{array}{l}\text { Added } \\
\text { Noise (dB) }\end{array}$ & $\begin{array}{l}\text { Total Noise } \\
\text { (dB) }\end{array}$ \\
\hline Noise Type - 1 & ID_Spat - 1 & 4.250 & 33.860 & 38.110 \\
\hline Noise Type - 1 & ID_Spat - 2 & 5.865 & 34.965 & 40.831 \\
\hline Noise Type - 1 & ID_Spat - 3 & 4.038 & 33.689 & 37.726 \\
\hline Noise Type - 1 & ID_Spat - 4 & 6.357 & 34.315 & 40.672 \\
\hline Noise Type - 1 & ID_Spat - 5 & 1.775 & 33.648 & 35.422 \\
\hline Noise Type - 1 & ID_Spat - 6 & 2.814 & 33.642 & 36.456 \\
\hline Noise Type - 1 & ID_Spat - 7 & 16.436 & 35.586 & 52.021 \\
\hline Noise Type - 1 & ID_Spat - 8 & 1.387 & 34.000 & 35.387 \\
\hline Noise Type - 1 & ID_Spat - 9 & 5.829 & 33.903 & 39.732 \\
\hline Noise Type - 1 & ID_Spat - 10 & 114.262 & 45.715 & 159.976 \\
\hline Noise Type - 2 & ID_Spat - 11 & 5.950 & 31.544 & 37.494 \\
\hline Noise Type - 2 & ID_Spat - 12 & 5.865 & 31.474 & 37.339 \\
\hline Noise Type - 2 & ID_Spat - 13 & 4.038 & 31.568 & 35.605 \\
\hline Noise Type - 2 & ID_Spat - 14 & 6.357 & 31.600 & 37.957 \\
\hline Noise Type - 2 & ID_Spat - 15 & 1.420 & 31.699 & 33.119 \\
\hline Noise Type - 2 & ID_Spat - 16 & 4.925 & 31.682 & 36.607 \\
\hline Noise Type - 2 & ID_Spat - 17 & 38.350 & 32.114 & 70.464 \\
\hline Noise Type - 2 & ID_Spat - 18 & 4.854 & 31.472 & 36.327 \\
\hline Noise Type - 2 & ID_Spat - 19 & 3.886 & 31.556 & 35.441 \\
\hline Noise Type - 2 & ID_Spat - 20 & 159.966 & 36.839 & 196.805 \\
\hline Noise Type - 3 & ID_Spat - 21 & 5.100 & 12.183 & 17.283 \\
\hline Noise Type - 3 & ID_Spat - 22 & 5.865 & 11.919 & 17.785 \\
\hline Noise Type - 3 & ID_Spat - 23 & 4.845 & 12.080 & 16.925 \\
\hline Noise Type - 3 & ID_Spat - 24 & 10.595 & 12.063 & 22.658 \\
\hline Noise Type - 3 & ID_Spat - 25 & 2.485 & 12.253 & 14.737 \\
\hline Noise Type - 3 & ID_Spat - 26 & 1.407 & 11.980 & 13.387 \\
\hline oise Type - 3 & ID_Spat - 27 & 38.350 & 12.638 & 50.988 \\
\hline Noise Type - 3 & ID_Spat - 28 & 2.774 & 11.853 & 14.627 \\
\hline Noise Type - 3 & ID_Spat - 29 & 5.829 & 12.014 & 17.842 \\
\hline Noise Type - 3 & ID_Spat - 30 & 137.114 & 24.586 & 161.700 \\
\hline Noise Type - 4 & ID_Spat - 31 & 2.550 & 32.927 & 35.476 \\
\hline Noise Type - 4 & ID_Spat - 32 & 20.529 & 27.979 & 48.507 \\
\hline Noise Type - 4 & ID_Spat - 33 & 1.615 & 32.483 & 34.099 \\
\hline Noise Type - 4 & ID_Spat - 34 & 6.357 & 31.017 & 37.374 \\
\hline Noise Type - 4 & ID_Spat - 35 & 1.065 & 33.839 & 34.904 \\
\hline Noise Type - 4 & ID_Spat - 36 & 2.814 & 32.195 & 35.009 \\
\hline Noise Type - 4 & ID_Spat - 37 & 21.914 & 25.968 & 47.882 \\
\hline Noise Type - 4 & ID_Spat - 38 & 3.467 & 31.406 & 34.874 \\
\hline Noise Type - 4 & ID_Spat - 39 & 6.800 & 31.742 & 38.542 \\
\hline Noise Type - 4 & ID_Spat - 40 & 159.966 & 29.772 & 189.738 \\
\hline
\end{tabular}

TABLE. II. NOISE DETECTION AND REMOVAL FOR SPATIAL IMAGES

\begin{tabular}{|c|c|c|c|c|}
\hline Data Item & $\begin{array}{l}\text { Total } \\
\text { Noise } \\
\text { (dB) }\end{array}$ & $\begin{array}{l}\text { Reduced } \\
\text { Noise (dB) } \\
\text { By } \\
\text { Removal } \\
\text { Method - 1 } \\
{[11]}\end{array}$ & $\begin{array}{l}\text { Reduced } \\
\text { Noise (dB) } \\
\text { By } \\
\text { Removal } \\
\text { Method - 2 } \\
{[12]}\end{array}$ & $\begin{array}{l}\text { Reduced } \\
\text { Noise }(d B) \\
\text { By Proposed } \\
\text { Method }\end{array}$ \\
\hline ID_Spat - 1 & 38.110 & 0.011 & 0.948 & 2.033 \\
\hline ID_Spat - 2 & 40.831 & 0.012 & 2.002 & 3.632 \\
\hline ID_Spat - 3 & 37.726 & 0.011 & 0.847 & 2.028 \\
\hline ID_Spat - 4 & 40.672 & 0.011 & 1.550 & 2.025 \\
\hline ID_Spat - 5 & 35.422 & 0.011 & 0.527 & 2.471 \\
\hline ID_Spat - 6 & 36.456 & 0.011 & 0.751 & 2.093 \\
\hline ID_Spat - 7 & 52.021 & 0.012 & 2.032 & 2.380 \\
\hline ID_Spat - 8 & 35.387 & 0.011 & 0.728 & 2.218 \\
\hline ID_Spat - 9 & 39.732 & 0.011 & 1.066 & 2.059 \\
\hline ID_Spat - 10 & 159.976 & 0.015 & 6.931 & 2.991 \\
\hline ID_Spat - 11 & 37.494 & 0.009 & 4.947 & 1.972 \\
\hline ID_Spat - 12 & 37.339 & 0.009 & 5.395 & 3.733 \\
\hline ID_Spat - 13 & 35.605 & 0.009 & 4.939 & 1.961 \\
\hline ID_Spat - 14 & 37.957 & 0.009 & 5.115 & 1.998 \\
\hline ID_Spat - 15 & 33.119 & 0.009 & 4.888 & 2.673 \\
\hline ID_Spat - 16 & 36.607 & 0.009 & 4.905 & 2.292 \\
\hline ID_Spat - 17 & 70.464 & 0.009 & 5.243 & 2.100 \\
\hline ID_Spat - 18 & 36.327 & 0.009 & 4.892 & 2.297 \\
\hline ID_Spat - 19 & 35.441 & 0.009 & 4.974 & 2.072 \\
\hline ID_Spat - 20 & 196.805 & 0.011 & 7.658 & 3.163 \\
\hline ID_Spat - 21 & 17.283 & 0.004 & 2.118 & 1.051 \\
\hline ID_Spat - 22 & 17.785 & 0.004 & 2.635 & 2.496 \\
\hline ID_Spat - 23 & 16.925 & 0.004 & 2.091 & 1.033 \\
\hline ID_Spat - 24 & 22.658 & 0.004 & 2.385 & 1.134 \\
\hline ID_Spat - 25 & 14.737 & 0.004 & 1.960 & 1.358 \\
\hline ID_Spat - 26 & 13.387 & 0.004 & 1.987 & 1.178 \\
\hline ID_Spat - 27 & 50.988 & 0.004 & 2.498 & 1.578 \\
\hline ID_Spat - 28 & 14.627 & 0.003 & 1.936 & 1.175 \\
\hline ID_Spat - 29 & 17.842 & 0.004 & 2.119 & 1.088 \\
\hline ID_Spat - 30 & 161.700 & 0.008 & 6.224 & 3.038 \\
\hline ID_Spat - 31 & 35.476 & 0.010 & 6.399 & 2.034 \\
\hline ID_Spat - 32 & 48.507 & 0.008 & 5.771 & 5.326 \\
\hline ID_Spat - 33 & 34.099 & 0.009 & 6.275 & 1.846 \\
\hline ID_Spat - 34 & 37.374 & 0.009 & 6.123 & 1.816 \\
\hline ID_Spat - 35 & 34.904 & 0.010 & 6.564 & 2.808 \\
\hline ID_Spat - 36 & 35.009 & 0.009 & 6.236 & 2.343 \\
\hline ID_Spat - 37 & 47.882 & 0.008 & 5.024 & 1.634 \\
\hline ID_Spat - 38 & 34.874 & 0.009 & 6.115 & 2.361 \\
\hline ID_Spat - 39 & 38.542 & 0.009 & 6.175 & 2.315 \\
\hline ID_Spat - 40 & 189.738 & 0.010 & 6.566 & 3.445 \\
\hline
\end{tabular}


TABLE. III. NoISE Reduction Percentage ANALysis For SPATIAL IMAGES

\begin{tabular}{|c|c|c|c|}
\hline Data Item & $\begin{array}{l}\text { Reduction \% by } \\
\text { Method - } 1\end{array}$ & $\begin{array}{l}\text { Reduction \% by } \\
\text { Method }-2\end{array}$ & $\begin{array}{l}\text { Reduction \% by } \\
\text { Proposed } \\
\text { Method }\end{array}$ \\
\hline ID_Spat - 1 & 88.819 & 86.362 & 83.515 \\
\hline ID_Spat - 2 & 85.606 & 80.731 & 76.740 \\
\hline ID_Spat - 3 & 89.267 & 87.052 & 83.921 \\
\hline ID_Spat - 4 & 84.342 & 80.559 & 79.392 \\
\hline ID_Spat - 5 & 94.958 & 93.503 & 88.013 \\
\hline ID_Spat - 6 & 92.250 & 90.220 & 86.538 \\
\hline ID_Spat - 7 & 68.384 & 64.501 & 63.831 \\
\hline ID_Spat -8 & 96.049 & 94.023 & 89.814 \\
\hline ID_Spat - 9 & 85.302 & 82.648 & 80.148 \\
\hline ID_Spat - 10 & 28.567 & 24.243 & 26.706 \\
\hline ID_Spat - 11 & 84.107 & 70.938 & 78.871 \\
\hline ID_Spat - 12 & 84.267 & 69.843 & 74.294 \\
\hline ID_Spat - 13 & 88.634 & 74.787 & 83.151 \\
\hline ID_Spat - 14 & 83.228 & 69.777 & 77.987 \\
\hline ID_Spat - 15 & 95.685 & 80.954 & 87.642 \\
\hline ID_Spat - 16 & 86.521 & 73.148 & 80.286 \\
\hline ID_Spat - 17 & 45.562 & 38.136 & 42.595 \\
\hline ID_Spat - 18 & 86.612 & 73.171 & 80.314 \\
\hline ID_Spat - 19 & 89.010 & 75.001 & 83.191 \\
\hline ID_Spat - 20 & 18.713 & 14.827 & 17.111 \\
\hline ID_Spat - 21 & 70.472 & 58.236 & 64.415 \\
\hline ID_Spat - 22 & 66.999 & 52.206 & 52.989 \\
\hline ID_Spat - 23 & 71.350 & 59.019 & 65.269 \\
\hline ID_Spat - 24 & 53.224 & 42.712 & 48.235 \\
\hline ID_Spat - 25 & 83.116 & 69.840 & 73.926 \\
\hline ID_Spat - 26 & 89.463 & 74.644 & 80.693 \\
\hline ID_Spat - 27 & 24.779 & 19.887 & 21.692 \\
\hline ID_Spat - 28 & 81.013 & 67.798 & 73.000 \\
\hline ID_Spat - 29 & 67.313 & 55.456 & 61.235 \\
\hline ID_Spat - 30 & 15.200 & 11.356 & 13.326 \\
\hline ID_Spat - 31 & 92.786 & 74.776 & 87.078 \\
\hline ID_Spat - 32 & 57.662 & 45.782 & 46.699 \\
\hline ID_Spat - 33 & 95.236 & 76.860 & 89.848 \\
\hline ID_Spat - 34 & 82.967 & 66.608 & 78.131 \\
\hline ID_Spat-35 & 96.921 & 78.143 & 88.905 \\
\hline ID_Spat - 36 & 91.935 & 74.150 & 85.269 \\
\hline ID_Spat - 37 & 54.217 & 43.740 & 50.821 \\
\hline ID_Spat - 38 & 90.032 & 72.524 & 83.287 \\
\hline ID_Spat - 39 & 82.333 & 66.336 & 76.349 \\
\hline ID_Spat - 40 & 15.686 & 12.231 & 13.875 \\
\hline
\end{tabular}

As the reduced noise levels are concerns, much reductions can be observed by the first methods [XX]. However, the other image integrity-based factors are highly compromised. The details are furnished and discussed further in the sections of the work. Thirdly, the noise reduction percentage by these three methods are also analysed here [Table III].

The results are also visualized graphically here [Fig. 4].

Further, the image information loss analysis is carried out. One of the most prominent measure of the information loss from the images are size of the image apart from the pixel intensity and scale density. The size comparison after the noise reduction is furnished here [Table IV].

The results are also visualized graphically here [Fig. 5].

The results are also visualized graphically here [Fig. 6].

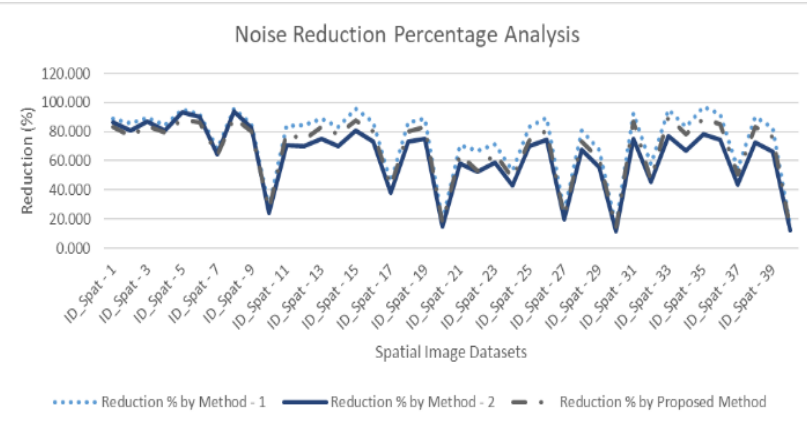

Fig. 4. Noise Level Reduction Percentage Analysis.

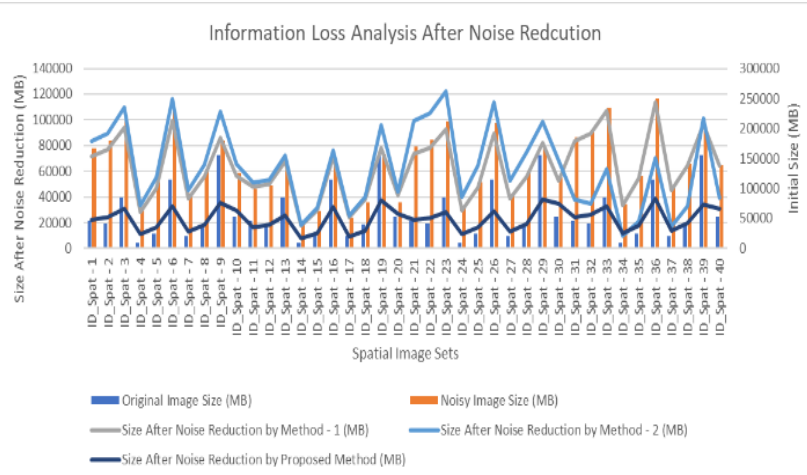

Fig. 5. Information Loss Percentage Analysis is Formulated [Table V]

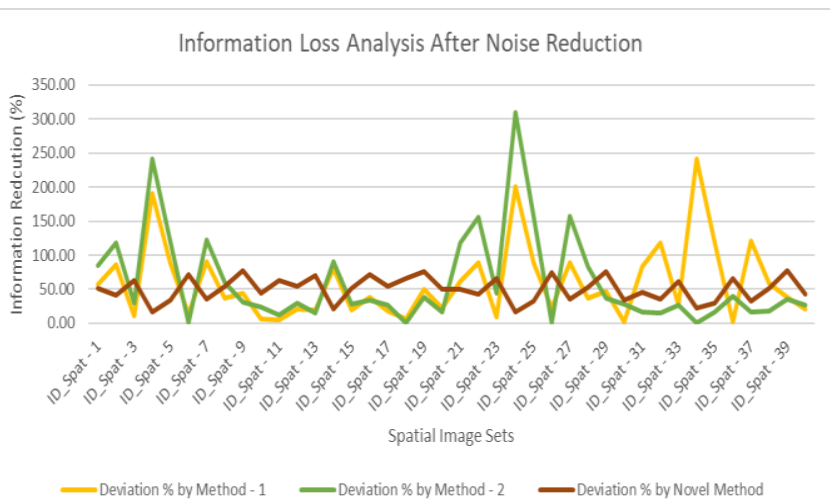

Fig. 6. Information Percentage Loss Analysis. 
TABLE. IV. SIZE ANALYSIS AFTER THE NOISE REDUCTION FOR SPATIAL IMAGES

\begin{tabular}{|c|c|c|c|c|c|}
\hline Data Item & $\begin{array}{l}\text { Origin } \\
\text { al } \\
\text { Image } \\
\text { Size } \\
\text { (MB) }\end{array}$ & $\begin{array}{l}\text { Noisy } \\
\text { Image } \\
\text { Size } \\
\text { (MB) }\end{array}$ & $\begin{array}{l}\text { Size After } \\
\text { Noise } \\
\text { Reduction } \\
\text { by } \\
\text { Method - } \\
1 \text { (MB) }\end{array}$ & $\begin{array}{l}\text { Size After } \\
\text { Noise } \\
\text { Reduction } \\
\text { by } \\
\text { Method - } \\
2 \text { (MB) }\end{array}$ & $\begin{array}{l}\text { Size After } \\
\text { Noise } \\
\text { Reduction } \\
\text { by } \\
\text { Proposed } \\
\text { Method } \\
\text { (MB) } \\
\end{array}$ \\
\hline ID_Spat - 1 & 45437 & 167331 & 71693 & 83712 & 22351 \\
\hline ID_Spat - 2 & 41130 & 178854 & 76705 & 89544 & 24447 \\
\hline ID_Spat - 3 & 84698 & 214678 & 94117 & 109470 & 30794 \\
\hline ID_Spat - 4 & 9755 & 66685 & 28429 & 33312 & 11351 \\
\hline ID_Spat - 5 & 24968 & 109963 & 46808 & 54912 & 16531 \\
\hline ID_Spat - 6 & 115202 & 228475 & 99408 & 116482 & 32575 \\
\hline ID_Spat - 7 & 20457 & 91513 & 38863 & 45497 & 13082 \\
\hline ID_Spat - 8 & 40564 & 128879 & 55745 & 64985 & 18351 \\
\hline ID_Spat - 9 & 155164 & 179606 & 85817 & 106752 & 35199 \\
\hline ID_Spat - 10 & 53071 & 125578 & 56409 & 66033 & 29640 \\
\hline ID_Spat - 11 & 45437 & 99249 & 47950 & 51110 & 16721 \\
\hline ID_Spat - 12 & 41130 & 104508 & 49877 & 53377 & 18594 \\
\hline ID_Spat - 13 & 84698 & 133089 & 67833 & 72264 & 25537 \\
\hline ID_Spat - 14 & 9755 & 38124 & 17532 & 18605 & 7775 \\
\hline ID_Spat - 15 & 24968 & 63250 & 29653 & 31839 & 12001 \\
\hline ID_Spat - 16 & 115202 & 144451 & 71066 & 76103 & 31859 \\
\hline ID_Spat - 17 & 20457 & 51769 & 24214 & 25793 & 9468 \\
\hline ID_Spat - 18 & 40564 & 76102 & 38241 & 40258 & 13885 \\
\hline ID_Spat - 19 & 155164 & 157122 & 78464 & 95699 & 37598 \\
\hline ID_Spat - 20 & 53071 & 77273 & 41105 & 44247 & 26842 \\
\hline ID_Spat - 21 & 45437 & 169599 & 73438 & 99399 & 22528 \\
\hline ID_Spat - 22 & 41130 & 180482 & 78018 & 105462 & 23567 \\
\hline ID_Spat - 23 & 84698 & 212141 & 92933 & 122483 & 28543 \\
\hline ID_Spat - 24 & 9755 & 67926 & 29310 & 39980 & 11391 \\
\hline ID_Spat - 25 & 24968 & 110082 & 47055 & 64150 & 16718 \\
\hline ID_Spat - 26 & 115202 & 208493 & 89502 & 113856 & 28939 \\
\hline ID_Spat - 27 & 20457 & 90795 & 38538 & 52554 & 13168 \\
\hline ID_Spat - 28 & 40564 & 128375 & 55702 & 74616 & 19211 \\
\hline ID_Spat - 29 & 155164 & 168397 & 82160 & 98588 & 38230 \\
\hline ID_Spat - 30 & 53071 & 117449 & 52395 & 68099 & 35066 \\
\hline ID_Spat - 31 & 45437 & 184563 & 83364 & 38048 & 24542 \\
\hline ID_Spat - 32 & 41130 & 197409 & 89594 & 35099 & 26516 \\
\hline ID_Spat - 33 & 84698 & 234286 & 106951 & 61869 & 32634 \\
\hline ID_Spat - 34 & 9755 & 73556 & 33317 & 9857 & 11937 \\
\hline ID_Spat - 35 & 24968 & 120899 & 54596 & 20914 & 17454 \\
\hline ID_Spat - 36 & 115202 & 249398 & 113641 & 70122 & 38501 \\
\hline ID_Spat - 37 & 20457 & 100838 & 45352 & 17157 & 13953 \\
\hline ID_Spat - 38 & 40564 & 141868 & 64307 & 33048 & 19995 \\
\hline ID_Spat - 39 & 155164 & 195594 & 96521 & 101160 & 33836 \\
\hline ID_Spat - 40 & 53071 & 138009 & 64324 & 39223 & 30793 \\
\hline
\end{tabular}

TABLE. V. SizE ANALYSIS AFTER THE NOISE REDUCTION FOR SPATIAL IMAGES

\begin{tabular}{|c|c|c|c|c|c|}
\hline Data Item & $\begin{array}{l}\text { Origin } \\
\text { al } \\
\text { Image } \\
\text { Size } \\
\text { (MB) }\end{array}$ & $\begin{array}{l}\text { Noisy } \\
\text { Image } \\
\text { Size } \\
\text { (MB) }\end{array}$ & $\begin{array}{l}\text { Deviatio } \\
\text { n \% by } \\
\text { Method } \\
-1\end{array}$ & $\begin{array}{l}\text { Deviation } \\
\text { \% by } \\
\text { Method - } 2\end{array}$ & $\begin{array}{l}\text { Deviation } \\
\% \text { by } \\
\text { Novel } \\
\text { Method }\end{array}$ \\
\hline ID_Spat - 1 & 45437 & 167331 & 57.79 & 84.24 & 50.81 \\
\hline ID_Spat - 2 & 41130 & 178854 & 86.49 & 117.71 & 40.56 \\
\hline ID_Spat - 3 & 84698 & 214678 & 11.12 & 29.25 & 63.64 \\
\hline ID_Spat - 4 & 9755 & 66685 & 191.43 & 241.49 & 16.36 \\
\hline ID_Spat - 5 & 24968 & 109963 & 87.47 & 119.93 & 33.79 \\
\hline ID_Spat - 6 & 115202 & 228475 & 13.71 & 1.11 & 71.72 \\
\hline ID_Spat - 7 & 20457 & 91513 & 89.97 & 122.40 & 36.05 \\
\hline ID_Spat - 8 & 40564 & 128879 & 37.42 & 60.20 & 54.76 \\
\hline ID_Spat - 9 & 155164 & 179606 & 44.69 & 31.20 & 77.31 \\
\hline ID_Spat - 10 & 53071 & 125578 & 6.29 & 24.42 & 44.15 \\
\hline ID_Spat - 11 & 45437 & 99249 & 5.53 & 12.49 & 63.20 \\
\hline ID_Spat - 12 & 41130 & 104508 & 21.27 & 29.78 & 54.79 \\
\hline ID_Spat - 13 & 84698 & 133089 & 19.91 & 14.68 & 69.85 \\
\hline ID_Spat - 14 & 9755 & 38124 & 79.72 & 90.72 & 20.30 \\
\hline ID_Spat - 15 & 24968 & 63250 & 18.76 & 27.52 & 51.93 \\
\hline ID_Spat - 16 & 115202 & 144451 & 38.31 & 33.94 & 72.35 \\
\hline ID_Spat - 17 & 20457 & 51769 & 18.37 & 26.08 & 53.72 \\
\hline ID_Spat - 18 & 40564 & 76102 & 5.73 & 0.75 & 65.77 \\
\hline ID_Spat - 19 & 155164 & 157122 & 49.43 & 38.32 & 75.77 \\
\hline ID_Spat - 20 & 53071 & 77273 & 22.55 & 16.63 & 49.42 \\
\hline ID_Spat - 21 & 45437 & 169599 & 61.63 & 118.76 & 50.42 \\
\hline ID_Spat - 22 & 41130 & 180482 & 89.69 & 156.41 & 42.70 \\
\hline ID_Spat - 23 & 84698 & 212141 & 9.72 & 44.61 & 66.30 \\
\hline ID_Spat - 24 & 9755 & 67926 & 200.46 & 309.84 & 16.77 \\
\hline ID_Spat - 25 & 24968 & 110082 & 88.46 & 156.93 & 33.04 \\
\hline ID_Spat - 26 & 115202 & 208493 & 22.31 & 1.17 & 74.88 \\
\hline ID_Spat - 27 & 20457 & 90795 & 88.39 & 156.90 & 35.63 \\
\hline ID_Spat - 28 & 40564 & 128375 & 37.32 & 83.95 & 52.64 \\
\hline ID_Spat - 29 & 155164 & 168397 & 47.05 & 36.46 & 75.36 \\
\hline ID_Spat - 30 & 53071 & 117449 & 1.27 & 28.32 & 33.93 \\
\hline ID_Spat - 31 & 45437 & 184563 & 83.47 & 16.26 & 45.99 \\
\hline ID_Spat - 32 & 41130 & 197409 & 117.83 & 14.66 & 35.53 \\
\hline ID_Spat - 33 & 84698 & 234286 & 26.27 & 26.95 & 61.47 \\
\hline ID_Spat - 34 & 9755 & 73556 & 241.54 & 1.05 & 22.37 \\
\hline ID_Spat - 35 & 24968 & 120899 & 118.66 & 16.24 & 30.09 \\
\hline ID_Spat - 36 & 115202 & 249398 & 1.36 & 39.13 & 66.58 \\
\hline ID_Spat - 37 & 20457 & 100838 & 121.69 & 16.13 & 31.79 \\
\hline ID_Spat - 38 & 40564 & 141868 & 58.53 & 18.53 & 50.71 \\
\hline ID_Spat - 39 & 155164 & 195594 & 37.79 & 34.80 & 78.19 \\
\hline ID_Spat - 40 & 53071 & 138009 & 21.20 & 26.09 & 41.98 \\
\hline
\end{tabular}


Hence, it is natural to realize that, the information loss is the least by the proposed method. Further, as the spatial dataset does not only concern the image data, rather also the text data. Hence, this algorithm also analyses the missing value detection and outlier removal results.

The missing value analysis is carried out for the complete spatial dataset. However, only few furnished here [Table VI].

The results are also visualized graphically here [Fig. 7].

The outlier analysis is carried out for the complete spatial dataset. However, only few are furnished here [Table VII].

TABLE. VI. Missing VALUe Detection ANALYsis

\begin{tabular}{|l|l|l|l|l|}
\hline Data Item & $\begin{array}{l}\text { Number of } \\
\text { Data Items }\end{array}$ & $\begin{array}{l}\text { Number of } \\
\text { Missing Values }\end{array}$ & $\begin{array}{l}\text { Detected } \\
\text { Missing } \\
\text { Values }\end{array}$ & $\begin{array}{l}\text { Accuracy } \\
(\%)\end{array}$ \\
\hline ID_Spat - 1 & 400 & 8 & 7 & 87.50 \\
\hline ID_Spat - 2 & 393 & 9 & 9 & 100.00 \\
\hline ID_Spat - 3 & 387 & 7 & 7 & 100.00 \\
\hline ID_Spat - 4 & 310 & 5 & 5 & 100.00 \\
\hline ID_Spat - 5 & 320 & 5 & 5 & 100.00 \\
\hline ID_Spat - 6 & 337 & 5 & 4 & 80.00 \\
\hline ID_Spat - 7 & 301 & 10 & 6 & 60.00 \\
\hline ID_Spat - 8 & 369 & 7 & 6 & 85.71 \\
\hline ID_Spat - 9 & 322 & 9 & 7 & 77.78 \\
\hline ID_Spat - 10 & 329 & 7 & 7 & 100.00 \\
\hline
\end{tabular}

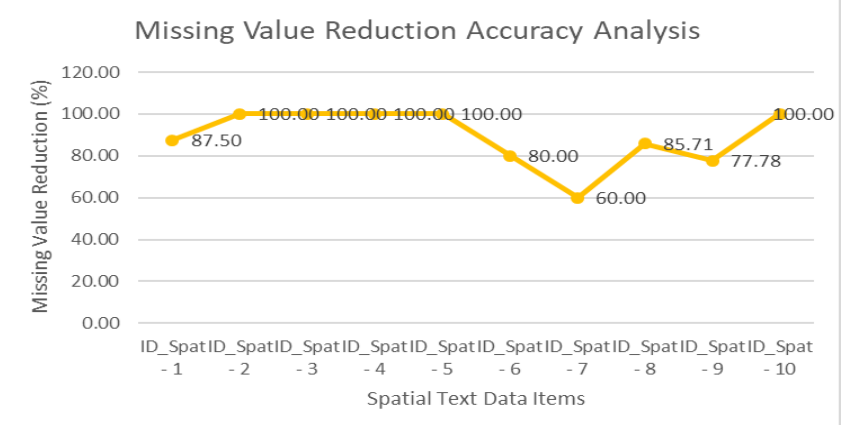

Fig. 7. Missing Value Replacement Accuracy Analysis.

TABLE. VII. OutLIER VALue Detection ANALYsis

\begin{tabular}{|l|l|l|l|l|}
\hline Data Item & $\begin{array}{l}\text { Number of } \\
\text { Data Items }\end{array}$ & $\begin{array}{l}\text { Number of } \\
\text { Missing } \\
\text { Values }\end{array}$ & $\begin{array}{l}\text { Detected } \\
\text { Missing } \\
\text { Values }\end{array}$ & $\begin{array}{l}\text { Accuracy } \\
(\%)\end{array}$ \\
\hline ID_Spat - 1 & 400 & 4 & 4 & 100.00 \\
\hline ID_Spat - 2 & 393 & 8 & 6 & 75.00 \\
\hline ID_Spat - 3 & 387 & 7 & 5 & 71.43 \\
\hline ID_Spat - 4 & 310 & 4 & 4 & 100.00 \\
\hline ID_Spat - 5 & 320 & 7 & 7 & 100.00 \\
\hline ID_Spat - 6 & 337 & 6 & 5 & 83.33 \\
\hline ID_Spat - 7 & 301 & 5 & 5 & 100.00 \\
\hline ID_Spat - 8 & 369 & 5 & 4 & 80.00 \\
\hline ID_Spat - 9 & 322 & 4 & 4 & 100.00 \\
\hline ID_Spat - 10 & 329 & 7 & 6 & 85.71 \\
\hline
\end{tabular}

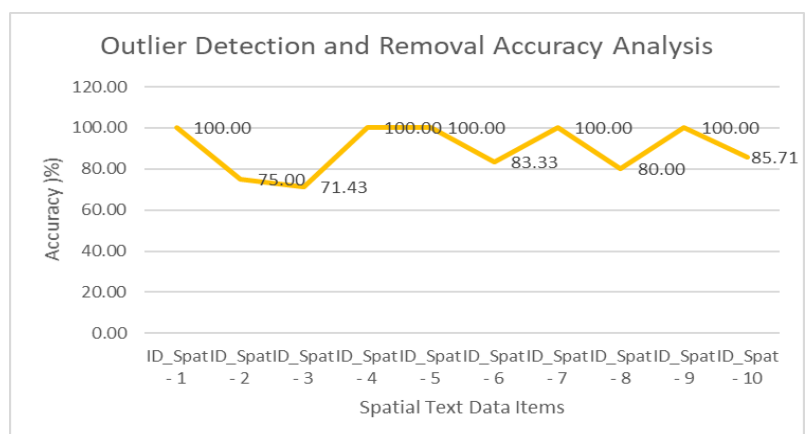

Fig. 8. Outlier Detection and Replacement Accuracy Analysis.

The results are also visualized graphically here [Fig. 8].

Henceforth, with the detailed analysis of the results obtained from the proposed algorithms, in the next section of this work, the comparative analysis is furnished.

\section{COMPARATIVE ANALYSIS}

Although, the step by step comparisons are carried out with highly benchmarked parallel research outcomes, in the previous section of this work, the summarized comparative analysis is carried out here [Table VIII].

The improvements over the existing algorithms are notable and the reasons are elaborated here.

Firstly, instead quite a few complexes mean of plasma normalization are constructing on arrangement preserving transformations homeomorphisms and also diffeomorphisms given that they take sleek sub manifolds effortlessly throughout conversion. Diffeomorphisms are created inside today's area of computational anatomy predicted on diffeomorphic leaks, additionally referred to as diffeomorphic mapping. But such transformations by way of diffeomorphic aren't additive, even though they produce a set with work article and behaving non-linearly to the graphics by the way of team actions.

Secondly, any process for spectral imaging, allowing qualitative and qualitative characterization of their air and also of this outside. These dimensions may be properly utilized for atmospheric gases, the dimension of these concentrations and identifications of outside substances and unambiguous direct, then the mission of their participation of signs that were blended.

TABLE. VIII. SUMMARIZED COMPARATIVE ANALYSIS

\begin{tabular}{|l|l|l|l|l|l|}
\hline $\begin{array}{l}\text { Method } \\
\text { Name }\end{array}$ & $\begin{array}{l}\text { Noise } \\
\text { Reduction } \\
\text { Percentage } \\
\text { Mean (\%) }\end{array}$ & $\begin{array}{l}\text { Image } \\
\text { Information } \\
\text { Loss } \\
\text { Percentage } \\
\text { Mean (\%) }\end{array}$ & $\begin{array}{l}\text { Missing } \\
\text { Value } \\
\text { Detection } \\
\text { and } \\
\text { Reduction } \\
\text { Accuracy } \\
\text { Mean (\%) }\end{array}$ & $\begin{array}{l}\text { Outlier } \\
\text { Detection } \\
\text { and } \\
\text { Reduction } \\
\text { Accuracy } \\
\text { Mean (\%) }\end{array}$ & $\begin{array}{l}\text { Model } \\
\text { Complexity }\end{array}$ \\
\hline $\begin{array}{l}\text { A. Ertürk } \\
\text { et al [11] }\end{array}$ & 73.96 & 59.51 & 59.21 & 57.55 & High \\
\hline $\begin{array}{l}\text { D. } \\
\text { Çeşmeci et } \\
\text { al. [12] }\end{array}$ & 63.67 & 60.65 & 61.75 & 62.62 & Moderate \\
\hline $\begin{array}{l}\text { Proposed } \\
\text { Method }\end{array}$ & 68.01 & 50.31 & 89.10 & 89.55 & Low \\
\hline
\end{tabular}


Third, in machine-learning and predictive optimization, loss works for classifications are all computationally viable loss purposes representing the cost covered inaccuracy of predictions from classification issues as issues of identifying that category a specific monitoring belongs to.

Finally, there will be to unmixing a method always to undo the procedure that is blending. Ordinarily, of blending 2 types are all supposed: nonlinear and linear. Vertical blending like being level models that the earth and episode sun onto the earth results in the substance to digitize a certain sum of their episode energy right back into the detector. Just about every pixel is described as a sum of the vitality slabs of substances. Just about every material contributes to the monitoring of this sensor within a mode that is favourable. A conservation of electricity restriction is detected inducing this mixture's weights to amount to a in addition to having the favourable.

Henceforth, after the detailed discussion on the comparative analysis, this work presence the final research conclusion in the next section of the work.

\section{CONCLUSION}

Automation in the road accident prediction is highly dependent on the road conditions. Thus, correct detections of the road conditions are one of the most important aspects. The road data or the spatial road information is highly prune to the noise. Failing to justify the de-noising process of the spatial data with respect to the image intensity or the information loss from the parallel research outcomes, this work proposes a novel adaptive moments-based image de-noising methods with the use of adaptive intensity calculations for image segments. Further in order to also de-noise the endmember data items, extracted from the spatial data, this work introduces two other methods as adaptive logistic estimation and corrective logistic estimation for the de-noising purpose. The work results in nearly $90 \%$ accuracy of de-nosing process. This work can be considered as a newer benchmark for denoising the spatial road data for making the further processing highly accurate.

\section{REFERENCES}

[1] D. A. Landgrebe. "Signal Theory Method in Multispectral Remote Sensing",Hoboken, NJ, USA:Wiley.2003.

[2] J. M. Bioucas-Dias. Hyperspectral unmixing overview: Geometrical statistical and sparse regression-based approaches", IEEE J. Sel. Topics Appl. Earth Observ. Remote Sens. 2012 vol.,no.2,pp.354-379.

[3] N. Keshava J. F. Mustard. "Spectral unmixing", IEEE Signal Process. Mag. 2002 vol. 19, no. 1, pp. 44-57, Jan.

[4] M. E. Winter. "N-FINDR: An algorithm for fast autonomous spectral endmember determination in hyperspectral data", P roc. SPIE,1999. vol. 3753, pp. 266-277.

[5] J.M.P.Nascimento,,J.M.Bioucas-Dias.-"Vertex-component, Analysis: A fast algorithm tounmi- hyperspectral data “, IEEE Trans.Geosci.Remote Sens. 2005 vol. 43, no. 4, pp. 898-910, Apr.

[6] J. Li, J. Bioucas-Dias. Minimum volume simplex Analysis: A fast algorithm to unmix hyperspectral Data ,Proc. IEEE Int. Geosci. Remote Symp.2008 (IGARSS),vol.3,pp.250-253.

[7] Alp Ertürk. Enhanced Unmixing-Based Hyperspectral Image Denoising Using Spatial Preprocessing, IEEE Journal of Selected Topics in Applied Earth Observations and Remote Sensing, Volume: 8, Issue: 6. 2015.

[8] M. Zortea, A. Plaza. Spatial preprocessing for endmember extraction, IEEE Trans. Geosci.RemoteSens.,vol.47,no.8,pp.2679-2693.2009.

[9] G. Martin, A. Plaza. Region-based Spatial preprocessing for endmember extraction and spectral unmixing. IEEE Trans. Geosci. Remote Sens., vol.8, no. 4,pp.745-749. 2011.

[10] G. Martin, A. Plaza. Spatial-spectral preprocessing prior to endmember identification and unmixing of remotely sensed hyperspectral data, 2012. IEEE-J.Sel. opics Appl. Earthbserv.RemoteSens.,vol.5,no.2,pp.380395.

[11] Ertürk, D. Çeşmeci, M. K. Güllü, D.Gerçek, and S. Ertürk. "Integrating Anomaly detection to spatial preprocessing for endmember extraction of Hyperspectralimages".2013,Proc.IEEE,Geosci.RemoteSens .Symp.(IGA RSS),pp.1087-1090.

[12] A.Ertürk, D. Çeşmeci. M. K. Güllü D. Gerçek, and S. Ertürk. "Endmember extraction guided by anomalies and homogeneous regions forhyperspectralimages",2014,IEEE.Sel.opicsAppl.EarthObserv.Remote Sens.,vol.7,no.8,pp.30-3639,Aug.

[13] C. Li, L. Wang. Incorporating spatial information in spectral unmixing: A review .RemoteSens.Environ.vol.149,pp.70-87,Jun.2015.

[14] Dataset: Indian Surface Maintenance Authority Image Samples. D. A. Landgrebe. "Signal Theory Method in Multispectral Remote Sensing",Hoboken, NJ, USA:Wiley.2003.M. Young, The Technical Writer's Handbook. Mill Valley, CA: University Science, 1989. 